\title{
TR-LIF LIFETIME MEASUREMENTS AND HFR+CPOL CALCULATIONS OF RADIATIVE PARAMETERS IN VANADIUM ATOM (V I)
}

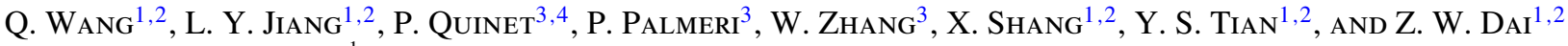 \\ ${ }^{1}$ College of Physics, Jilin University, Changchun 130012, China; dai@jlu.edu.cn \\ ${ }^{2}$ Key Lab of Coherent Light, Atomic and Molecular Spectroscopy, Ministry of Education, Changchun 130012, China \\ ${ }^{3}$ Astrophysique et Spectroscopie, Université de Mons_UMONS, 20 Place du Parc, B-7000 Mons, Belgium; Pascal.quinet@ umons.ac.be \\ ${ }^{4}$ IPNAS, Université de Liège, B-4000 Liège, Belgium \\ Received 2014 January 16; accepted 2014 March 3; published 2014 April 4
}

\begin{abstract}
Radiative lifetimes of 79 levels belonging to the $3 d^{3} 4 s 4 p, 3 d^{4} 4 p, 3 d^{3} 4 s 5 p, 3 d^{4} 5 p$, and $3 d^{3} 4 s 4 d$ configurations of V I with energy from $26,604.807$ to $46,862.786 \mathrm{~cm}^{-1}$ have been measured using time-resolved laser-induced fluorescence (TR-LIF) spectroscopy in laser-produced plasma. The lifetime values reported in this paper are in the range of 3.3-494 ns, and the uncertainties of these measurements are within $\pm 10 \%$. A good agreement was obtained with previous data. HFR+CPOL calculations have been performed and used to combine the calculated branching fractions with the available experimental lifetimes to determine semi-empirical transition probabilities for $784 \mathrm{~V}$ I transitions.
\end{abstract}

Key words: atomic data - methods: laboratory: atomic - techniques: spectroscopic

Online-only material: machine-readable table

\section{INTRODUCTION}

The determination of elemental abundances and their patterns in stellar atmospheres has recently challenged the accuracy of the oscillator strengths used in stellar spectrum modeling. More precisely, an accuracy of the order of $\sim 0.05$ dex in differential abundances (and therefore also in $\log g f$ ) is needed to detect the effect of planet formation in the stellar atmospheres (Meléndez et al. 2009; Ramírez et al. 2014). These effects are marked by a $\sim 20 \%$ depletion of refractory elements with respect to the volatile elements. Vanadium $(Z=$ $23)$ is a refractory element that belongs to the iron group. Due to its complex electronic structure related to an open $3 d$ subshell and a relatively high cosmic abundance, e.g., $\varepsilon_{V}=$ $\log \left(N_{V} / N_{\mathrm{H}}\right)+12=3.93 \pm 0.08$ in the Sun (Asplund et al. 2009), many lines of V I and V II are observed in solar and stellar spectra. Experimental oscillator strengths are essential for the determination of elemental abundances but direct measurements of oscillator strengths are difficult (Den Hartog et al. 2011; Larsson 1994). A reliable and convenient method for obtaining experimental oscillator strengths is through the combination of measured radiative lifetimes with accurate branching fractions (BFs). Hence, the measurements of lifetimes and BFs are of great significance.

Roberts et al. (1973) measured radiative lifetimes for 14 levels of $V_{I}$ in the energy range $21,963.45-40,535.70 \mathrm{~cm}^{-1}$ using the beam-foil technique. Rudolph \& Helbig (1982) measured the lifetimes of nine levels of $\mathrm{V}$ I using a selective pulsed laser excitation in an atomic beam produced by a special cage formed with tungsten and vanadium wire. Doerr et al. (1985) reported 12 lifetimes of VI levels by selective laser excitation of an atomic beam produced by thermal evaporation of vanadium metal. Later on, Whaling et al. (1985) combined their time-resolved laser-induced fluorescence (TR-LIF) lifetimes obtained for 39 odd-parity levels with their Fourier transform spectroscopic BFs to determine absolute transition probabilities for $208 \mathrm{~V}$ I lines in the spectral range 318-1640 nm. More recently, Xu et al. (2006) measured 10 lifetimes of V I in the energy range $30,635.59-41,928.48 \mathrm{~cm}^{-1}$ using the TR-LIF experimental method.

The lifetimes of highly excited VI energy levels $(E>$ $27,000 \mathrm{~cm}^{-1}$ ) available in the literature are relatively scarce. Moreover, there are still differences between some measurements that go beyond their error bars. These have motivated the present TR-LIF lifetime measurements of the 79 highly excited levels ranging from $26,604.807$ to $46,862.786 \mathrm{~cm}^{-1}$. In addition, HFR+CPOL calculations have been carried out to provide BFs in order to obtain the absolute semi-empirical radiative rates for $784 \mathrm{~V}$ I lines.

\section{EXPERIMENTAL SETUP}

The time-resolved laser spectroscopic methods can selectively excite specific atomic levels using tunable lasers and reduce the influence of cascade effects on the measured radiative lifetimes. This technique has already proven its reliability and efficiency and has been used many times with success by many groups around the world (Doerr et al. 1985; Den Hartog et al. 2011; Xu et al. 2006). The experimental setup used for the lifetime measurement is similar to that described in our previous papers (Feng et al. 2010; Zhang et al. 2010), and so only a brief description is given here.

A $532 \mathrm{~nm}$ Nd:YAG laser with a $8 \mathrm{~ns}$ pulse duration emitted with $10 \mathrm{~Hz}$ repetition rate and an energy of $5-10 \mathrm{~mJ}$ was focused on a rotating vanadium foil in a vacuum chamber through a $30 \mathrm{~cm}$ focal-length lens to produce laser-induced plasma. The latter, employed as a free-atom source, supplied sufficient numbers of $\mathrm{V}$ atoms in the ground and in some metastable states. In order to obtain a tunable excitation pulse, another Nd:YAG laser with characteristics similar to the ablation pulse was used to pump a dye laser (Sirah Cobra-Stretch) in which DCM dye operated. The second-order harmonic of the dye laser produced by a beta barium borate (BBO) crystal was focused into a stimulated Raman scattering cell with $\mathrm{H}_{2}$ at 15 bars to obtain the first-order Stokes component of Raman shifting. The third-order harmonic of the dye laser was obtained with the use of two BBO crystals and a retarding plate. Thus, the wavelength 


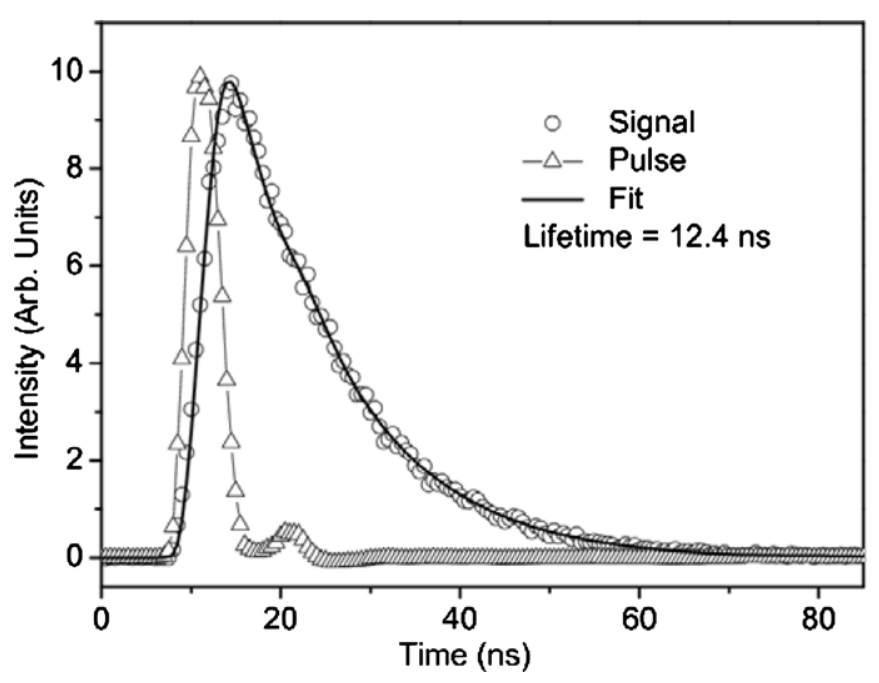

Figure 1. Typical fluorescence decay curve of the $42,245.489 \mathrm{~cm}^{-1}$ level of V I with the fitted convolution curve between the laser pulse and an exponential.

of the excitation pulse is tunable in the range $210-380 \mathrm{~nm}$. The excitation pulse with about 6 ns duration was sent horizontally into the vacuum chamber about $8 \mathrm{~mm}$ above the sample to excite free $\mathrm{V}$ atoms. The delay time between the ablation and excitation pulses was adjusted by a digital delay generator (SRS DG535). The fluorescence emitted from the measured levels was imaged by a fused silica lens and focused into a grating monochromator $(f=10 \mathrm{~cm})$ for dispersion and then detected by a microchannel plate photomultiplier tube (Hamamatsu R3809U-58) with a 163 ps rise time. A $2.5 \mathrm{GHz}$ digital oscilloscope (Tektronix DPO7254) was used to register and average the transient signals from the detector.

In order to wash out quantum beats produced by the Earth's magnetic field and reduce the recombination background from the plasma, an appropriate magnetic field of about $100 \mathrm{G}$ produced by a pair of Helmholtz coils in the direction along the horizontal component of Earth's magnetic field was employed during the experiment (Wang et al. 2013).

\section{LIFETIME MEASUREMENTS}

In the present experiment, the single-step excitation scheme was used. The targeted odd-parity levels of V I were populated from the ground state and some metastable states ranging from 0 to $17,182.073 \mathrm{~cm}^{-1}$. Also, some excited evenparity levels could be reached using the metastable odd levels $3 d^{3}\left({ }^{4} F\right) 4 s 4 p\left({ }^{3} P\right) z^{6} G^{0}{ }_{3 / 2-13 / 2}$ ranging from $16,361.489$ to $17,136.538 \mathrm{~cm}^{-1}$. Their lifetimes were estimated to range from a fraction of a millisecond to a few seconds using the model described in the next section.

In the measurements, in order to avoid simultaneous excitation of other levels from different metastable states, the exciting wavelength has been carefully chosen from all available excitation pathways. Moreover, the excitation level under study was confirmed by verifying that the observed fluorescence wavelengths were related to this level. The radiation trapping effect, the collisional effect, the flight-out-of-view effect, and the saturation effect which could possibly contribute to the systematic errors of the experimental results have been paid careful attention. The details for checking and eliminating these effects were the same as described in our previous paper (Feng et al. 2011).

To obtain a good signal-to-noise ratio, more than 1000 shots were averaged for each fluorescence decay curve. More than

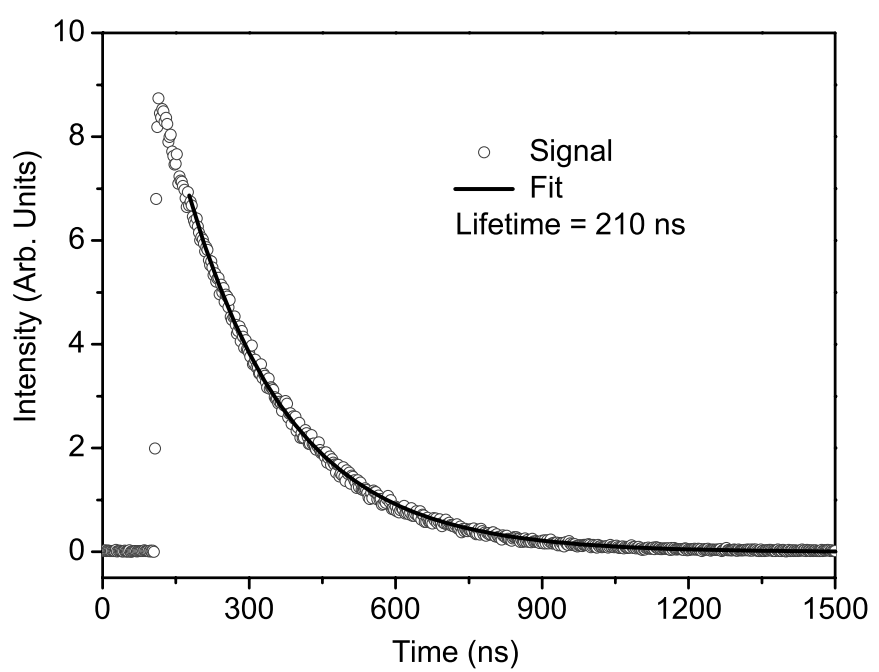

Figure 2. Typical fluorescence decay curve of the $31317.440 \mathrm{~cm}^{-1}$ level of V I with an exponential fit for lifetime evaluation.

10 curves under different conditions were recorded for each level, and the average value of the lifetimes evaluated from these curves was taken as the final lifetime. The lifetime values shorter than $50 \mathrm{~ns}$ were evaluated by fitting the experimental fluorescence decay curve to a convolution of the recorded laser excitation pulse and a pure exponential function. A typical fluorescence decay curve for the $42,245.489 \mathrm{~cm}^{-1}$ level with a 12.4(0.9) ns lifetime is shown in Figure 1. For the other longer-lived levels, a least-squares exponential fit procedure was performed to obtain lifetime values. The starting point for the fitting procedure must be chosen carefully to avoid the influence of excitation pulse. A typical fluorescence decay curve for the $31,317.440 \mathrm{~cm}^{-1}$ level with a $210(15) \mathrm{ns}$ lifetime obtained by an exponential fit is shown in Figure 2. One can see from Figures 1 and 2 that the fluorescence decay curves were fitted well, and each signal had a good signal-to-noise ratio.

\section{HFR+CPOL CALCULATIONS}

The Hartree-Fock with relativistic corrections method (HFR) originally developed by Cowan (1981) in which we have included core-polarization (CPOL) effects (see, e.g., Quinet et al. 1999) was used to calculate the lifetimes, the BFs, and the transition probabilities in V I. The intravalence correlation was accounted for by considering the following configurations in the configuration interaction (CI) expansions: $3 d^{3} 4 s^{2}+$ $3 d^{3} 4 s 5 s+3 d^{3} 4 s 6 s+3 d^{4} 4 s+3 d^{4} 5 s+3 d^{4} 6 s+3 d^{5}+3 d^{4} 4 d+$ $3 d^{4} 5 d+3 d^{4} 6 d+3 d^{3} 4 s 4 d+3 d^{3} 4 s 5 d+3 d^{3} 4 s 6 d+3 d^{3} 4 p^{2}+$ $3 d^{3} 4 d^{2}+3 d^{3} 4 f^{2}+3 d^{3} 4 p 4 f+3 d^{3} 4 s 4 p^{2}+3 d^{2} 4 s^{2} 4 d$ for the even parity and $3 d^{4} 4 p+3 d^{4} 5 p+3 d^{4} 6 p+3 d^{3} 4 s 4 p+3 d^{3} 4 s 5 p+$ $3 d^{3} 4 s 6 p+3 d^{4} 4 f+3 d^{3} 4 s 4 f+3 d^{3} 4 d 4 f+3 d^{2} 4 s^{2} 4 p+3 d^{2} 4 p^{3}$ for the odd parity. The core-valence correlation was modeled with a polarization potential and a correction to the electric dipole transition operator in which a V IV $3 d^{2}$ ionic core was considered with a dipole polarizability of 2.09 a.u. (Fraga et al. 1976) and a cut-off radius equal to the HFR average radius $\langle r\rangle$ of the $3 d$ orbital, i.e., 1.36 a.u.

A 0.8 scaling factor has been applied to the Slater integrals according to a well-established practice in order to consider the effect of far-interacting configurations not explicitly included in the multiconfiguration expansions.

Some radial integrals, considered to be free parameters, were then adjusted with a least-squares optimization program 


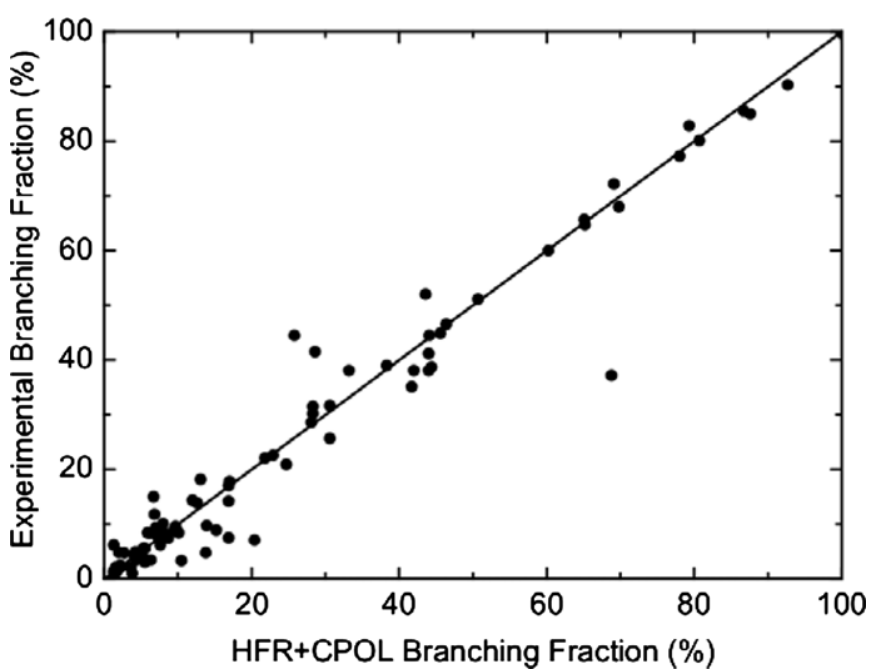

Figure 3. Comparison between the HFR+CPOL branching fractions obtained in this work and those measured by Whaling et al. (1985). The values affected by strong cancellation effects are not plotted (see the text).

minimizing the discrepancies between the calculated Hamiltonian eigenvalues and the experimental energy levels taken from the most recent term analysis of Thorne et al. (2011). More precisely, we followed the fitting procedure described by Thorne et al. (2011) and used their radial parameters as starting values, i.e., the average energies $\left(E_{\mathrm{av}}\right)$, the direct coulomb interaction integrals within the $3 d^{N}$ (with $\left.N=3,4\right)$ cores $\left(F^{2}(3 d 3 d)\right.$ and $F^{4}(3 d 3 d)$ ) and the effective interaction parameters $(\alpha$ and $\beta$ ) were fitted as well as the CI Slater integrals between the two odd-parity configurations $3 d^{4} 4 p$ and $3 d^{3} 4 s 4 p\left(R^{2}(3 d 3 d, 3 d 4 s)\right.$, $R^{2}(3 d 4 p, 4 s 4 p)$, and $\left.R^{1}(3 d 4 p, 4 p 4 s)\right)$. The average deviations were $130 \mathrm{~cm}^{-1}$ for the even parity and $126 \mathrm{~cm}^{-1}$ for the odd parity.

Our HFR+CPOL lifetimes are compared with our TR-LIF measurements and previous experimental values in Table 1. Those for which the calculated line strengths of important decay channels (representing more than $10 \%$ of the radiative width) are affected by strong cancellation effects, i.e., their cancellation factors (CFs) as defined by Cowan (1981) are less than or equal to 0.05 , are marked with an asterisk. Some of the discrepancies between theory and experiment seen in this table can be explained by such a cancellation effect.

In Figure 3, a comparison between the BFs measured by Whaling et al. (1985) and those calculated in this work is shown. The BF values can be affected by strong cancellation effects, i.e., in the formula used for the determination of the theoretical branching fraction, $\mathrm{BF}_{i j}$,

$$
\mathrm{BF}_{i j}=A_{i j} \tau_{i}
$$

either the theoretical transition probability, $A_{i j}$, has a CF less than or equal to 0.05 or the theoretical lifetime is one of those marked with an asterisk in Table 1 or both. These values are not plotted. More precisely, 122 transitions were discarded in this way out of a total of 208. As one can see, except for a few points, a good agreement between both sets is obtained for the strong decay channels $(\mathrm{BF}>20 \%)$. Indeed strong cancellation effects can still play an indirect role in the few remaining disagreements seen in the figure. For instance, concerning the transition $3 d^{3}\left({ }^{4} F\right) 4 s 4 p$ $z^{6} D^{\mathrm{o}}{ }_{7 / 2}-3 d^{4}\left({ }^{5} D\right) 4 s a^{6} D_{7 / 2}$, although its $\mathrm{CF}$ value is greater than 0.05 , it has a HFR+CPOL BF-value equal to $68.8 \%(\mathrm{CF}=0.07)$ to be compared to the measurement of Whaling et al. (1985) of

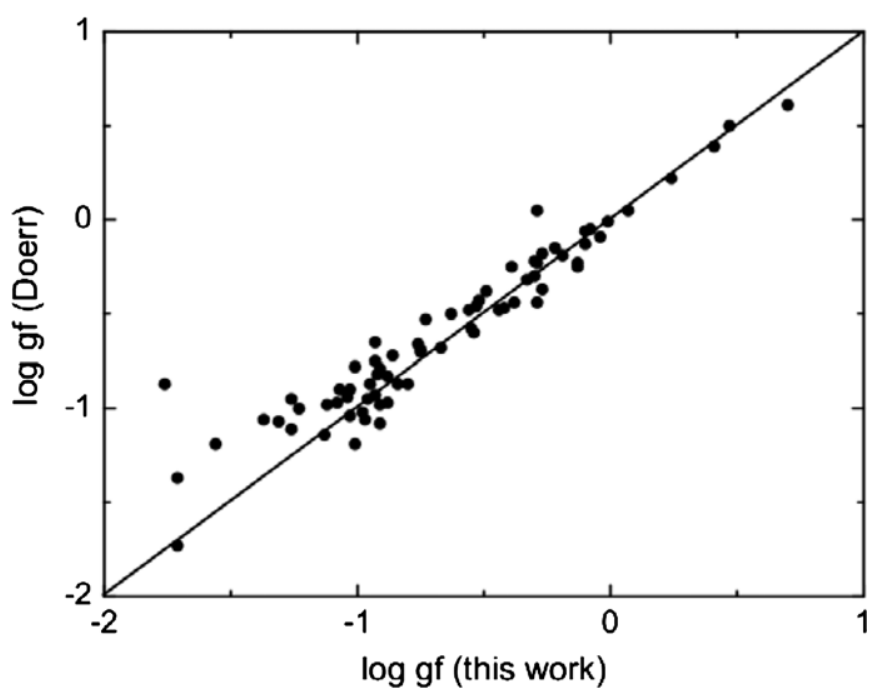

Figure 4. Comparison between the corrected $\log g f$ obtained in this work and the experimental $\log g f$ determined by Doerr et al. (1985) combining hook and emission measurements. Our values with $\mathrm{CF}<0.05$ are not plotted (see the text).

$37.2 \%$. This can be explained by the fact that the strongest decay branch from the $3 d^{3}\left({ }^{4} F\right) 4 s 4 p z^{6} D^{\circ}{ }_{7 / 2}$ (that decays to $3 d^{4}\left({ }^{5} D\right) 4 s$ $\left.a^{6} D_{5 / 2}\right)$, not plotted in the figure, with a measured BF of $41.0 \%$ (Whaling et al. 1985) is affected by a very strong cancellation effect $(\mathrm{CF}=0.01)$ giving rise to a weak calculated $\mathrm{BF}$ of $6.3 \%$ which affects the HFR+CPOL BF-values of all the other decay branches from that level through Equation (1).

In Table 2, the HFR+CPOL weighted transition probabilities $(g A)$, the HFR+CPOL weighted oscillator strengths in the logarithmic scale $(\log g f)$, and the HFR+CPOL BF are presented for the 784 strongest decay channels $(\mathrm{BF} \geqslant 1 \%)$ of the excited energy levels reported in Table 1 . Based on the good agreement between our HFR+CPOL BF values and measurements of Whaling et al. (1985), an improvement on the absolute scale of the radiative rates has been obtained by rescaling our calculated $A$ values (and consequently our calculated $f$ values). The corrected $A$ values have been extracted by combining the available experimental lifetimes (given just below the corresponding upper level of the transition) with the HFR+CPOL BF values using Equation (1). These corrected transition probabilities and oscillator strengths are given in the seventh and eighth columns of the table. In the last column, the $\mathrm{CF}$ is also reported. A $\mathrm{CF}$ value less than 0.05 indicates that the transition probability is affected by a strong cancellation effect and therefore should be taken with care.

The accuracy of our semi-empirical $f$ values can be further assessed by comparing with those determined by Doerr et al. (1985) combining hook and emission measurements. In Figure 4, we plot the $\log g f$ of Doerr et al. with respect to our corrected values discarding the 9 transitions (out of 81) for which the CF values are less than 0.05. A good agreement is found with an average difference between these two sets of 0.07 dex (the standard deviation being $0.16 \mathrm{dex}$ ). The remaining strong disagreement seen for the transition $3 d^{3}\left({ }^{4} P\right) 4 s 4 p\left({ }^{3} P\right)$ $w^{4} D^{o}{ }_{7 / 2}-3 d^{4}\left({ }^{5} D\right) 4 s a^{6} D_{7 / 2}$ (-0.87 in Doerr et al. compared to -1.76 in this work) is probably due to cancellation effects. Indeed, the CF value of 0.06 is close to threshold (0.05) and our HFR+CPOL lifetime of the upper level of that transition used to calculate the BF value is affected by strong cancellation effects (see Table 1). 
Table 1

Comparison between Theoretical and Experimental Lifetimes of V I Energy Levels

\begin{tabular}{|c|c|c|c|c|c|c|c|c|}
\hline \multicolumn{4}{|c|}{ Level $^{\mathrm{a}}$} & \multirow{2}{*}{$\begin{array}{l}\lambda_{\text {Exc. }} \\
(\mathrm{nm})\end{array}$} & \multirow{2}{*}{$\begin{array}{l}\lambda_{\text {Obs. }} \\
(\mathrm{nm})\end{array}$} & \multicolumn{3}{|c|}{ Lifetime (ns) } \\
\hline Configuration & Term & $J$ & $E\left(\mathrm{~cm}^{-1}\right)$ & & & This Expt. & Prev. Expt. & HFR \\
\hline \multirow[t]{5}{*}{$3 d^{3}\left({ }^{4} F\right) 4 s 4 p\left({ }^{3} P\right)$} & $z^{6} D^{\mathrm{o}}$ & $1 / 2$ & 18085.952 & & & & $390(40)^{\mathrm{b}}$ & $361^{*}$ \\
\hline & & $3 / 2$ & 18126.250 & & & & $395(40)^{b}$ & $389^{*}$ \\
\hline & & $5 / 2$ & 18198.091 & & & & $395(40)^{b}$ & $442^{*}$ \\
\hline & & $7 / 2$ & 18302.280 & & & & $385(40)^{\mathrm{b}}$ & 542 \\
\hline & & $9 / 2$ & 18438.044 & & & & $370(40)^{\mathrm{b}}$ & $492^{*}$ \\
\hline \multirow[t]{4}{*}{$3 d^{3}\left({ }^{4} F\right) 4 s 4 p\left({ }^{3} P\right)$} & $z^{4} D^{\mathrm{o}}$ & $1 / 2$ & 20606.467 & & & & $86(3)^{\mathrm{b}}$ & $87.9^{*}$ \\
\hline & & $3 / 2$ & 20687.769 & & & & $83(3)^{b}$ & $89.5^{*}$ \\
\hline & & $5 / 2$ & 20828.481 & & & & $89(3)^{\mathrm{b}}$ & $91.6^{*}$ \\
\hline & & $7 / 2$ & 21032.503 & & & & $92.5(3)^{\mathrm{b}}$ & $93.8^{*}$ \\
\hline \multirow[t]{4}{*}{$3 d^{3}\left({ }^{4} F\right) 4 s 4 p\left({ }^{3} P\right)$} & $z^{4} G^{\mathrm{o}}$ & $5 / 2$ & 21841.421 & & & & $183(3)^{\mathrm{b}}$ & $239^{*}$ \\
\hline & & $7 / 2$ & 21963.437 & & & & $179(3)^{\mathrm{b}} 12.4(1.9)^{\mathrm{c}}$ & $234^{*}$ \\
\hline & & $9 / 2$ & 22121.079 & & & & $174(3)^{\mathrm{b}}$ & $227^{*}$ \\
\hline & & $11 / 2$ & 22313.832 & & & & $168(3)^{\mathrm{b}} 12.0(1.8)^{\mathrm{c}}$ & $220^{*}$ \\
\hline \multirow[t]{5}{*}{$3 d^{3}\left({ }^{4} F\right) 4 s 4 p\left({ }^{3} P\right)$} & $z^{4} F^{\mathrm{o}}$ & $3 / 2$ & 23088.074 & & & & $145(3)^{\mathrm{b}}$ & $187^{*}$ \\
\hline & & $5 / 2$ & 23210.560 & & & & $132(3)^{\mathrm{b}}$ & $175^{*}$ \\
\hline & & $7 / 2$ & 23353.135 & & & & $128(4)^{b}$ & $173^{*}$ \\
\hline & & $9 / 2$ & 23519.872 & & & & $133(4)^{\mathrm{b}}$ & $174^{*}$ \\
\hline & & & & & & & $7.0(1.4)^{\mathrm{c}}$ & \\
\hline \multirow{4}{*}{$3 d^{4}\left({ }^{5} D\right) 4 p$} & $z^{4} P^{\mathrm{o}}$ & $1 / 2$ & 24770.673 & & & & $24(1)^{\mathrm{b}}$ & 23.1 \\
\hline & & $3 / 2$ & 24915.151 & & & & $24(1)^{b}$ & 23.1 \\
\hline & & & & & & & $9.5(2.0)^{\mathrm{d}}$ & \\
\hline & & $5 / 2$ & 25131.002 & & & & $25(1)^{b}$ & 23.0 \\
\hline \multirow[t]{8}{*}{$3 d^{4}\left({ }^{5} D\right) 4 p$} & $y^{4} F^{\mathrm{o}}$ & $3 / 2$ & 25930.544 & & & & $14.3(0.5)^{\mathrm{b}}$ & 13.3 \\
\hline & & & & & & & $14.3(1.0)^{\mathrm{e}}$ & \\
\hline & & $5 / 2$ & 26004.234 & & & & $14.4(0.5)^{b}$ & 13.3 \\
\hline & & & & & & & $15.0(1.1)^{\mathrm{e}}$ & \\
\hline & & $7 / 2$ & 26122.094 & & & & $17.6(0.5)^{\mathrm{b}}$ & 22.4 \\
\hline & & & & & & & $18.2(1.3)^{\mathrm{e}}$ & \\
\hline & & $9 / 2$ & 26171.918 & & & & $19.2(0.5)^{\mathrm{b}}$ & 14.6 \\
\hline & & & & & & & $21.0(1.5)^{\mathrm{e}}$ & \\
\hline \multirow[t]{3}{*}{$3 d^{3}\left({ }^{4} F\right) 4 s 4 p\left({ }^{3} P\right)$} & $z^{2} G^{\mathrm{o}}$ & $7 / 2$ & 26021.907 & & & & $67(3)^{b}$ & 26.1 \\
\hline & & & & & & & $71(5)^{\mathrm{e}}$ & \\
\hline & & $9 / 2$ & 26344.902 & & & & $49(3)^{\mathrm{b}}$ & 96.8 \\
\hline $3 d^{4}\left({ }^{5} D\right) 4 p$ & $y^{4} D^{\mathrm{o}}$ & $1 / 2$ & 26182.637 & & & & $12.3(0.5)^{\mathrm{b}}$ & 11.9 \\
\hline & & & & & & & $12.7(0.9)^{\mathrm{e}}$ & \\
\hline & & & & & & & $12.3(0.7)^{f}$ & \\
\hline & & $3 / 2$ & 26249.476 & & & & $12.3(0.5)^{\mathrm{b}}$ & 11.9 \\
\hline & & & & & & & $12.9(0.9)^{\mathrm{e}}$ & \\
\hline & & & & & & & $11.9(0.7)^{\mathrm{f}}$ & \\
\hline & & $5 / 2$ & 26352.634 & & & & $12.4(0.5)^{\mathrm{b}}$ & 12.1 \\
\hline & & & & & & & $13.3(0.9)^{\mathrm{e}}$ & \\
\hline & & & & & & & $12.4(0.7)^{\mathrm{f}}$ & \\
\hline & & $7 / 2$ & 26480.286 & & & & $12.5(0.5)^{\mathrm{b}}$ & 13.2 \\
\hline & & & & & & & $13.8(1.0)^{\mathrm{e}}$ & \\
\hline & & & & & & & $12.2(0.7)^{\mathrm{f}}$ & \\
\hline $3 d^{4}\left({ }^{5} D\right) 4 p$ & $y^{6} D^{\mathrm{o}}$ & $1 / 2$ & 26397.633 & & & & $7.7(0.5)^{b}$ & 7.06 \\
\hline & & & & & & & $8.0(0.4)^{\mathrm{f}}$ & \\
\hline & & $3 / 2$ & 26437.754 & & & & $7.8(0.5)^{\mathrm{b}}$ & 7.05 \\
\hline & & & & & & & $8.1(0.4)^{\mathrm{f}}$ & \\
\hline & & $5 / 2$ & 26505.953 & & & & $7.9(0.5)^{\mathrm{b}}$ & 7.04 \\
\hline & & & & & & & $7.9(0.4)^{\mathrm{f}}$ & \\
\hline & & $7 / 2$ & 26604.807 & 377.823 & 410 & $7.8(0.5)$ & $7.8(0.5)^{\mathrm{b}}$ & 7.05 \\
\hline & & & & & & & $8.0(0.4)^{\mathrm{f}}$ & \\
\hline & & $9 / 2$ & 26738.323 & & & & $7.9(0.5)^{b}$ & 7.16 \\
\hline & & & & & & & $7.8(0.4)^{\mathrm{f}}$ & \\
\hline & & & & & & & $9.3(1.4)^{\mathrm{c}}$ & \\
\hline $3 d^{3}\left({ }^{4} F\right) 4 s 4 p\left({ }^{3} P\right)$ & $z^{2} F^{\mathrm{o}}$ & $5 / 2$ & 27187.747 & 367.813 & 368 & 494(46) & & 617 \\
\hline $3 d^{3}\left({ }^{4} P\right) 4 s 4 p\left({ }^{3} P\right)$ & $x^{6} D^{\circ}$ & $1 / 2$ & 28313.626 & 353.187 & 382 & $36.4(2.5)$ & & 26.5 \\
\hline & & $3 / 2$ & 28368.753 & 354.216 & 381 & $36.5(2.6)$ & & 26.5 \\
\hline & & $5 / 2$ & 28462.177 & 355.382 & 380 & $37.7(2.6)$ & & 26.6 \\
\hline & & $7 / 2$ & 28595.637 & 356.600 & 379 & $38.7(2.7)$ & & 26.7 \\
\hline & & $9 / 2$ & 28768.142 & 377.976 & 379 & $39.7(2.8)$ & & 27.0 \\
\hline $3 d^{3}\left({ }^{4} P\right) 4 s 4 p\left({ }^{3} P\right)$ & $y^{6} P^{\mathrm{o}}$ & $3 / 2$ & 29202.790 & 369.133 & 371 & $6.4(0.4)$ & & 6.05 \\
\hline & & $5 / 2$ & 29296.430 & 368.417 & 371 & $6.9(0.5)$ & & 6.06 \\
\hline & & $7 / 2$ & 29418.119 & 367.675 & 371 & $7.0(0.5)$ & & 6.06 \\
\hline
\end{tabular}


Table 1

(Continued)

\begin{tabular}{|c|c|c|c|c|c|c|c|c|}
\hline \multicolumn{4}{|c|}{ Level $^{\mathrm{a}}$} & \multirow{2}{*}{$\begin{array}{l}\lambda_{\text {Exc. }} \\
(\mathrm{nm})\end{array}$} & \multirow{2}{*}{$\begin{array}{l}\lambda_{\text {Obs. }} \\
(\mathrm{nm})\end{array}$} & \multicolumn{3}{|c|}{ Lifetime (ns) } \\
\hline Configuration & Term & $J$ & $E\left(\mathrm{~cm}^{-1}\right)$ & & & This Expt. & Prev. Expt. & HFR \\
\hline \multirow[t]{3}{*}{$3 d^{3}\left({ }^{4} P\right) 4 s 4 p\left({ }^{3} P\right)$} & $y^{4} P^{0}$ & $1 / 2$ & 30021.627 & 358.830 & 462 & $49.3(3.5)$ & & $51.1^{*}$ \\
\hline & & $3 / 2$ & 30094.585 & 357.892 & 468 & $51.2(3.6)$ & & $52.9^{*}$ \\
\hline & & $5 / 2$ & 30120.823 & 359.590 & 464 & $55.8(3.9)$ & & $59.9^{*}$ \\
\hline \multirow[t]{4}{*}{$3 d^{3}\left({ }^{2} G\right) 4 s 4 p\left({ }^{3} P\right)$} & $y^{4} G^{\mathrm{o}}$ & $5 / 2$ & 30635.580 & 326.418 & 329 & $76.4(4.2)$ & $74.0(5)^{\mathrm{g}}$ & 8.69 \\
\hline & & $7 / 2$ & 30694.344 & 327.257 & 327 & & $87(6)^{\mathrm{g}}$ & 10.6 \\
\hline & & $9 / 2$ & 30771.732 & 328.425 & 328 & & $105(10)^{\mathrm{g}}$ & 13.8 \\
\hline & & $11 / 2$ & 30864.279 & 351.624 & 329 & $142(10)$ & & 19.0 \\
\hline \multirow[t]{4}{*}{$3 d^{3}\left({ }^{2} G\right) 4 s 4 p\left({ }^{3} P\right)$} & $x^{4} F^{\mathrm{o}}$ & $3 / 2$ & 31200.152 & 321.929 & 442 & $232(16)$ & & $185^{*}$ \\
\hline & & $5 / 2$ & 31229.014 & 323.566 & 444 & $208(15)$ & & $158^{*}$ \\
\hline & & $7 / 2$ & 31268.091 & 323.158 & 443 & $207(15)$ & & $166^{*}$ \\
\hline & & $9 / 2$ & 31317.440 & 325.050 & 442 & $210(15)$ & & 177 \\
\hline \multirow[t]{4}{*}{$3 d^{3}\left({ }^{4} F\right) 4 s 4 p\left({ }^{1} P\right)$} & $x^{4} G^{\mathrm{o}}$ & $5 / 2$ & 31397.822 & & & & $3.6(5)^{\mathrm{b}}$ & 4.23 \\
\hline & & $7 / 2$ & 31541.167 & & & & $3.6(5)^{\mathrm{b}}$ & 3.89 \\
\hline & & $9 / 2$ & 31721.780 & & & & $3.6(5)^{\mathrm{b}}$ & 3.59 \\
\hline & & $11 / 2$ & 31937.131 & & & & $3.6(5)^{\mathrm{b}}$ & 3.37 \\
\hline $3 d^{3}\left({ }^{2} P\right) 4 s 4 p\left({ }^{3} P\right)$ & $x^{4} D^{\mathrm{o}}$ & $3 / 2$ & 32456.581 & 309.414 & 417 & $90.6(6.3)$ & & $59.3^{*}$ \\
\hline \multirow[t]{2}{*}{$3 d^{3}\left({ }^{2} G\right) 4 s 4 p\left({ }^{3} P\right)$} & $y^{2} G^{\mathrm{o}}$ & $9 / 2$ & 33306.943 & 323.812 & 446 & $40.1(3.3)$ & & $117^{*}$ \\
\hline & & $7 / 2$ & 33360.280 & 323.253 & 445 & $59.4(4.2)$ & & $103^{*}$ \\
\hline $3 d^{3}\left({ }^{4} P\right) 4 s 4 p\left({ }^{3} P\right)$ & $w^{4} D^{\mathrm{o}}$ & $7 / 2$ & 34127.921 & 315.426 & 393 & $16.7(1.2)$ & & $14.0^{*}$ \\
\hline \multirow[t]{2}{*}{$3 d^{3}\left({ }^{4} F\right) 4 s 4 p\left({ }^{1} P\right)$} & $v^{4} D^{\mathrm{o}}$ & $3 / 2$ & 34537.299 & 309.433 & 383 & $18.6(1.3)$ & & 15.3 \\
\hline & & $5 / 2$ & 34619.598 & 309.519 & 386 & $11.5(0.8)$ & & 11.5 \\
\hline \multirow[t]{3}{*}{$3 d^{3}\left({ }^{2} D\right) 4 s 4 p\left({ }^{3} P\right)$} & $u^{4} D^{\mathrm{o}}$ & $1 / 2$ & 35013.259 & 376.832 & 285 & $17.4(1.2)$ & & 12.9 \\
\hline & & $3 / 2$ & 35092.518 & 374.819 & 572 & $18.7(1.3)$ & & 12.8 \\
\hline & & $5 / 2$ & 35224.990 & 377.227 & 283 & $21.4(1.5)$ & & 13.0 \\
\hline $3 d^{3}\left({ }^{4} P\right) 4 s 4 p\left({ }^{3} P\right)$ & $y^{4} S^{\mathrm{o}}$ & $3 / 2$ & 36408.402 & 357.202 & 376 & $10.2(0.7)$ & & $22.9^{*}$ \\
\hline \multirow[t]{2}{*}{$3 d^{4}\left(a^{3} F\right) 4 p$} & ${ }^{2} G^{\mathrm{o}}$ & $7 / 2$ & 36461.282 & 360.418 & 394 & $17.8(1.2)$ & & 16.3 \\
\hline & & $9 / 2$ & 36538.569 & 359.417 & 389 & $17.1(1.2)$ & & 17.0 \\
\hline $3 d^{4}\left(a^{3} P\right) 4 p$ & ${ }^{2} P^{\mathrm{o}}$ & $1 / 2$ & 36477.728 & 356.319 & 556 & $6.6(0.5)$ & & 26.9 \\
\hline & & $3 / 2$ & 36580.427 & 355.020 & 291 & $5.5(0.4)$ & & $36.7^{*}$ \\
\hline $3 d^{3}\left({ }^{2} D\right) 4 s 4 p\left({ }^{3} P\right)$ & $x^{4} P^{o}$ & $1 / 2$ & 36695.612 & 368.310 & 463 & $38.7(2.7)$ & & 25.9 \\
\hline & & $3 / 2$ & 36814.827 & 366.701 & 464 & $33.0(2.3)$ & & $22.4^{*}$ \\
\hline & & $5 / 2$ & 36611.845 & 370.717 & 355 & $43.8(3.1)$ & & $30.6^{*}$ \\
\hline $3 d^{3}\left({ }^{4} P\right) 4 s 4 p\left({ }^{3} P\right)$ & $x^{2} D^{\mathrm{o}}$ & $5 / 2$ & 36700.768 & 369.498 & 388 & $43.7(3.1)$ & & $42.9^{*}$ \\
\hline $3 d^{3}\left({ }^{2} H\right) 4 s 4 p\left({ }^{3} P\right)$ & $w^{4} G^{\mathrm{o}}$ & $7 / 2$ & 36822.878 & 354.054 & 385 & $37.5(3.0)$ & & 147 \\
\hline $3 d^{4}\left(a^{3} F\right) 4 p$ & $x^{2} F^{\mathrm{o}}$ & $5 / 2$ & 36766.041 & 353.484 & 271 & $5.7(0.4)$ & & 18.3 \\
\hline & & $7 / 2$ & 36925.893 & 352.767 & 369 & $9.5(0.7)$ & & 13.0 \\
\hline $3 d^{4}\left({ }^{3} H\right) 4 p$ & $v^{2} G^{\mathrm{o}}$ & $7 / 2$ & 37174.691 & 365.630 & 381 & $19.5(1.4)$ & & 16.8 \\
\hline & & $9 / 2$ & 37361.951 & 377.794 & 446 & $13.9(1.0)$ & & 11.6 \\
\hline $3 d^{4}\left({ }^{3} H\right) 4 p$ & $z^{2} I^{\mathrm{o}}$ & $11 / 2$ & 37530.314 & 378.362 & 444 & $20.2(1.4)$ & & 14.4 \\
\hline $3 d^{4}\left(a^{3} P\right) 4 p$ & $t^{4} D^{\mathrm{o}}$ & $1 / 2$ & 37757.309 & 264.849 & 354 & & $5.3(0.4)^{\mathrm{g}}$ & 5.23 \\
\hline & & $5 / 2$ & 37959.695 & 369.451 & 370 & $5.6(0.4)$ & & 7.21 \\
\hline & & $7 / 2$ & 38115.684 & 264.604 & 353 & & $5.6(0.4)^{\mathrm{g}}$ & 5.19 \\
\hline $3 d^{4}\left({ }^{3} H\right) 4 p$ & $x^{2} H^{\mathrm{o}}$ & $9 / 2$ & 38123.795 & 367.225 & 313 & $3.3(0.2)$ & & 14.2 \\
\hline & & $11 / 2$ & 38220.663 & 368.731 & 430 & $14.3(1.0)$ & & 13.9 \\
\hline $3 d^{4}\left({ }^{3} H\right) 4 p$ & $x^{4} H^{\mathrm{o}}$ & $7 / 2$ & 38245.829 & 365.586 & 422 & $8.3(0.6)$ & & 7.46 \\
\hline & & $11 / 2$ & 38405.067 & 366.240 & 426 & $8.1(0.6)$ & & 7.32 \\
\hline $3 d^{3}\left({ }^{2} H\right) 4 s 4 p\left({ }^{3} P\right)$ & $u^{2} G^{\mathrm{o}}$ & $9 / 2$ & 38529.830 & 364.575 & 423 & $8.5(0.6)$ & & $48.0^{*}$ \\
\hline $3 d^{4}\left(a^{3} P\right) 4 p$ & $w^{4} P^{\mathrm{o}}$ & $3 / 2$ & 39248.928 & 324.297 & 417 & 7.3(0.5) & & 6.13 \\
\hline & & $5 / 2$ & 39422.745 & 325.659 & 350 & $7.0(0.4)$ & & 5.79 \\
\hline $3 d^{4}\left(a^{3} F\right) 4 p$ & $u^{4} F^{\mathrm{o}}$ & $3 / 2$ & 39266.680 & 324.776 & 424 & $9.2(0.6)$ & $8.7(0.6)^{\mathrm{g}}$ & 6.72 \\
\hline & & $5 / 2$ & 39300.537 & 255.341 & 424 & & $9.4(0.7)^{\mathrm{g}}$ & 6.68 \\
\hline & & $7 / 2$ & 39341.784 & 256.289 & 423 & & $9.5(0.7)^{\mathrm{g}}$ & 6.76 \\
\hline & & $9 / 2$ & 39391.079 & 353.476 & 271 & $5.7(0.4)$ & $9.6(0.7)^{\mathrm{g}}$ & 6.89 \\
\hline $3 d^{4}\left(a^{3} F\right) 4 p$ & $s^{4} D^{\mathrm{o}}$ & $3 / 2$ & 39935.176 & 318.912 & 412 & $6.4(0.4)$ & & 5.25 \\
\hline $3 d^{4}\left({ }^{3} H\right) 4 p$ & $u^{4} G^{\mathrm{o}}$ & $5 / 2$ & 39962.150 & 320.037 & 344 & $27.6(1.1)$ & & 5.76 \\
\hline $3 d^{4}\left(a^{3} P\right) 4 p$ & $v^{2} D^{\mathrm{o}}$ & $5 / 2$ & 40119.218 & 317.051 & 396 & $14.0(0.5)$ & & 10.8 \\
\hline $3 d^{4}\left({ }^{3} G\right) 4 p$ & ${ }^{2} F^{o}$ & $5 / 2$ & 40325.790 & 313.977 & 405 & $9.7(0.4)$ & & 10.3 \\
\hline $3 d^{4}\left({ }^{3} G\right) 4 p$ & $t^{4} F^{\mathrm{o}}$ & $3 / 2$ & 41389.644 & 314.934 & 412 & $8.0(0.6)$ & & 8.09 \\
\hline & & $5 / 2$ & 41429.042 & 377.088 & 470 & $24.9(1.7)$ & & 7.88 \\
\hline & & $9 / 2$ & 41599.436 & 377.420 & 488 & $10.8(0.7)$ & & 7.21 \\
\hline $3 d^{4}\left({ }^{3} G\right) 4 p$ & $t^{2} G^{\mathrm{o}}$ & $7 / 2$ & 41436.610 & 377.540 & 300 & 130(13) & & 8.34 \\
\hline & & $9 / 2$ & 41539.185 & 376.815 & 326 & $10.1(0.7)$ & & 7.94 \\
\hline $3 d^{4}\left({ }^{3} G\right) 4 p$ & $t^{4} G^{\mathrm{o}}$ & $5 / 2$ & 41654.726 & 325.073 & 407 & $7.9(0.6)$ & & 6.79 \\
\hline & & & & 368.920 & 369 & 7.3(0.5) & & \\
\hline
\end{tabular}


Table 1

(Continued)

\begin{tabular}{|c|c|c|c|c|c|c|c|c|}
\hline \multicolumn{4}{|c|}{ Levela $^{\mathrm{a}}$} & \multirow{2}{*}{$\begin{array}{l}\lambda_{\text {Exc. }} \\
(\mathrm{nm})\end{array}$} & \multirow{2}{*}{$\begin{array}{l}\lambda_{\text {Obs. }} \\
(\mathrm{nm})\end{array}$} & \multicolumn{3}{|c|}{ Lifetime (ns) } \\
\hline Configuration & Term & $J$ & $E\left(\mathrm{~cm}^{-1}\right)$ & & & This Expt. & Prev. Expt. & HFR \\
\hline \multirow[t]{2}{*}{$3 d^{3}\left({ }^{4} P\right) 4 s 4 p\left({ }^{1} P\right)$} & $v^{4} P^{\mathrm{o}}$ & $1 / 2$ & 41751.931 & 310.489 & 377 & $7.4(0.5)$ & & $7.41^{*}$ \\
\hline & & $5 / 2$ & 42009.912 & 378.245 & 407 & $7.9(0.6)$ & & 7.76 \\
\hline \multirow[t]{2}{*}{$3 d^{4}\left({ }^{3} D\right) 4 p$} & $u^{2} F^{\mathrm{o}}$ & $5 / 2$ & 41950.393 & 364.489 & 322 & $12.0(1.2)$ & & 11.4 \\
\hline & & $7 / 2$ & 42020.903 & 371.511 & 399 & $7.5(0.5)$ & & 10.9 \\
\hline \multirow[t]{4}{*}{$3 d^{3}\left({ }^{4} P\right) 4 s 4 p\left({ }^{1} P\right)$} & $r^{4} D^{\mathrm{o}}$ & $1 / 2$ & 41928.473 & 238.501 & 309 & & $7.3(0.5)^{\mathrm{g}}$ & 8.10 \\
\hline & & $3 / 2$ & 41999.261 & 310.804 & 378 & $7.7(0.5)$ & & $8.08^{*}$ \\
\hline & & & & 378.398 & 308 & $7.7(0.5)$ & & \\
\hline & & $7 / 2$ & 42245.489 & 308.404 & 376 & $12.4(0.9)$ & & 9.08 \\
\hline \multirow[t]{2}{*}{$3 d^{4}\left({ }^{5} D\right) 5 p$} & ${ }^{6} F^{\mathrm{o}}$ & $5 / 2$ & 42172.123 & 378.102 & 371 & $42.8(3.0)$ & & $49.1^{*}$ \\
\hline & & $7 / 2$ & 42236.621 & 377.846 & 298 & $16.5(1.2)$ & & 84.8 \\
\hline $3 d^{3}\left({ }^{4} F\right) 4 \mathrm{~s} 5 p$ & ${ }^{6} D^{\mathrm{o}}$ & $7 / 2$ & 44026.568 & 370.761 & 339 & $10.8(1.0)$ & & $53.9^{*}$ \\
\hline $3 d^{3} 4 s\left({ }^{5} F\right) 4 d$ & $f^{6} P$ & $7 / 2$ & 44083.247 & 363.496 & 364 & $6.8(0.5)$ & & 7.25 \\
\hline $3 d^{3} 4 s\left({ }^{5} F\right) 4 d$ & ${ }^{6} F$ & $9 / 2$ & 44205.606 & 361.887 & 362 & $6.4(0.5)$ & & 6.57 \\
\hline $3 d^{3}\left({ }^{2} F\right) 4 s 4 p\left({ }^{3} P\right)$ & $p^{4} F^{\mathrm{o}}$ & $5 / 2$ & 45688.309 & 313.610 & 406 & $9.8(0.4)$ & & 10.4 \\
\hline \multirow[t]{3}{*}{$3 d^{3}\left({ }^{4} F\right) 4 s 5 p$} & $o^{4} D^{\mathrm{o}}$ & $3 / 2$ & 45762.309 & 312.884 & 403 & $10.8(0.8)$ & & 6.98 \\
\hline & & & & 312.975 & 401 & $10.9(0.8)$ & & \\
\hline & & $5 / 2$ & 45838.082 & 319.251 & 401 & $9.0(0.4)$ & & $6.86^{*}$ \\
\hline $3 d^{3}\left({ }^{2} F\right) 4 s 4 p\left({ }^{3} P\right)$ & $r^{4} G^{\mathrm{o}}$ & $5 / 2$ & 46052.702 & 217.142 & 317 & $9.5(0.7)$ & & 8.27 \\
\hline $3 d^{4}\left({ }^{3} D\right) 4 p$ & $t^{4} P^{\mathrm{o}}$ & $3 / 2$ & 46862.786 & 214.016 & 377 & $4.7(0.3)$ & & 3.89 \\
\hline
\end{tabular}

Notes. The excitation wavelength $\lambda_{\text {Exc. }}$ is in vacuum.

${ }^{a}$ Thorne et al. (2011).

${ }^{b}$ Whaling et al. (1985).

${ }^{\mathrm{c}}$ Roberts et al. (1973).

${ }^{\mathrm{d}}$ Childs et al. (1979)

e Rudolph \& Helbig (1982).

${ }^{\mathrm{f}}$ Doerr et al. (1985).

${ }^{\mathrm{g}} \mathrm{Xu}$ et al. (2006).

* Strong cancellation effects on lifetime (see the text).

Table 2

Transition Probabilities $(g A)$, Oscillator Strengths ( $\log g f)$ and Branching Fractions (BF) for the Strongest Decay Channels Depopulating the Levels for which the Lifetime has been Measured in V I

\begin{tabular}{|c|c|c|c|c|c|c|c|c|}
\hline Upper Level $^{\mathrm{a}}$ & Lower Level $^{\mathrm{a}}$ & $\begin{array}{c}\lambda^{\mathrm{a}} \\
(\mathrm{nm})\end{array}$ & $\begin{array}{c}g A \\
\left(\mathrm{~s}^{-1}\right)\end{array}$ & $\log g f$ & $\begin{array}{l}\mathrm{BF} \\
(\%)\end{array}$ & $\begin{array}{c}g A_{\text {cor }}{ }^{b} \\
\left(\mathrm{~s}^{-1}\right)\end{array}$ & $\log g f_{\text {cor }}^{\mathrm{b}}$ & $\mathrm{CF}^{\mathrm{c}}$ \\
\hline 18085.952 (о) $1 / 2$ & 2112.282 (e) $1 / 2$ & 625.8571 & $9.00 \mathrm{E}+05$ & -2.28 & 16.2 & $8.33 E+05$ & -2.31 & 0.04 \\
\hline$\tau=390(40)^{\mathrm{d}} \mathrm{ns}$ & 2153.221 (e) $3 / 2$ & 627.4652 & $4.63 \mathrm{E}+06$ & -1.56 & 83.6 & $4.29 \mathrm{E}+06$ & -1.60 & 0.08 \\
\hline 18126.250 (о) $3 / 2$ & 2112.282 (e) $1 / 2$ & 624.2822 & $2.89 \mathrm{E}+06$ & -1.77 & 28.1 & $2.85 \mathrm{E}+06$ & -1.78 & 0.04 \\
\hline \multirow[t]{2}{*}{$\tau=395(40)^{\mathrm{d}} \mathrm{ns}$} & 2153.221 (e) $3 / 2$ & 625.8822 & $5.02 \mathrm{E}+05$ & -2.53 & 4.9 & $4.95 \mathrm{E}+05$ & -2.54 & 0.05 \\
\hline & 2220.156 (e) $5 / 2$ & 628.5160 & $6.88 \mathrm{E}+06$ & -1.39 & 66.9 & $6.78 \mathrm{E}+06$ & -1.40 & 0.08 \\
\hline 18198.091 (о) $5 / 2$ & 2153.221 (e) $3 / 2$ & 623.0798 & $2.71 \mathrm{E}+06$ & -1.80 & 20.0 & $3.03 \mathrm{E}+06$ & -1.75 & 0.02 \\
\hline \multirow[t]{2}{*}{$\tau=395(40)^{\mathrm{d}} \mathrm{ns}$} & 2220.156 (e) $5 / 2$ & 625.6900 & $4.35 \mathrm{E}+06$ & -1.59 & 32.1 & $4.87 \mathrm{E}+06$ & -1.54 & 0.07 \\
\hline & 2311.369 (e) $7 / 2$ & 629.2824 & $6.50 \mathrm{E}+06$ & -1.41 & 47.9 & $7.27 \mathrm{E}+06$ & -1.36 & 0.08 \\
\hline 18302.280 (о) $7 / 2$ & 2220.156 (e) $5 / 2$ & 621.6364 & $9.34 \mathrm{E}+05$ & -2.27 & 6.3 & $1.31 \mathrm{E}+06$ & -2.12 & 0.01 \\
\hline$\tau=385(40)^{\mathrm{d}} \mathrm{ns}$ & 2311.369 (e) $7 / 2$ & 625.1823 & $1.02 \mathrm{E}+07$ & -1.23 & 68.8 & $1.43 \mathrm{E}+07$ & -1.08 & 0.07 \\
\hline
\end{tabular}

Notes. The energy levels are labeled by their values, parities ("e" for even and "o" for odd), and total quantum number $J$. The wavelengths are determined from the energy levels of this reference by the Ritz formula and are given in air above $200 \mathrm{~nm}$.

${ }^{a}$ Thorne et al. (2011).

${ }^{b}$ Determined by a combination of the experimental lifetime (reported below the upper level) and the HFR+CPOL BF.

${ }^{c}$ Cancellation factor (CF) as defined in Cowan (1981). The transition probability for which the CF is less than 0.05 is affected by a strong cancellation effect and should be taken with caution.

${ }^{\mathrm{d}}$ Whaling et al. (1985).

e This work.

${ }^{\mathrm{f}}$ Xu et al. (2006).

(This table is available in its entirety in a machine-readable form in the online journal. A portion is shown here for guidance regarding its form and content.)

\section{RESULTS AND DISCUSSION}

In this paper, radiative lifetimes for $79 \mathrm{~V}_{\mathrm{I}}$ levels from $26,604.807$ to $46,862.786 \mathrm{~cm}^{-1}$ belonging to the configurations
$3 d^{3} 4 s 4 p, 3 d^{4} 4 p, 3 d^{3} 4 s 5 p, 3 d^{4} 5 p$, and $3 d^{3} 4 s 4 d$ have been measured and presented in Table 1. The quoted error bars consist of both the statistical uncertainties from different recordings and the systematic errors from the fitting processes. The lifetime 
values fall in the range from 3.3 to $494 \mathrm{~ns}$ with the uncertainties no more than $\pm 10 \%$. To the best of our knowledge, 75 lifetimes of $\mathrm{V}_{\mathrm{I}}$ levels were measured for the first time.

Our new measurements for the levels $y^{6} D^{\mathrm{o}}{ }_{7 / 2}, y^{4} G^{\mathrm{o}}{ }_{5 / 2}$, and $u^{4} F_{3 / 2}^{\mathrm{o}}$ are supported by previous TR-LIF values (Whaling et al. 1985; Doerr et al. 1985; Xu et al. 2006). An exception is the case of the level $u^{4} F_{9 / 2}^{\mathrm{o}}$ for which our experimental value is slightly closer to our HFR+CPOL calculation than the measurement of $\mathrm{Xu}$ et al. (2006).

Although our HFR+CPOL calculation supports our measurements and previous experimental lifetimes in several instances, important disagreements still remain that cannot be explained by cancellation effects. A noticeable example is given by the $y^{4} G^{\mathrm{o}}$ multiplet for which the experimental lifetimes range from 74.0(5.0) ns to $142(10) \mathrm{ns}$ and the HFR+CPOL values are an order of magnitude shorter, ranging from $8.69 \mathrm{~ns}$ to $19.0 \mathrm{~ns}$. In that case, line blends seem unlikely to be the cause of the observed differences because the experimental lifetimes of all the levels of this multiplet are of the same order of magnitude. Actually, a strong intermediate coupling mixing occurs for this term between the basis vectors $3 d\left({ }^{2} G\right) 4 s 4 p{ }^{4} G^{\mathrm{o}}$ (with contributions ranging from $57 \%$ for $J=5 / 2$ to $73 \%$ for $J=11 / 2$ ) and $3 d\left({ }^{4} F\right) 4 s 4 p{ }^{4} G^{\circ}$ (with contributions ranging from $20 \%$ for $J=$ $11 / 2$ to $31 \%$ for $J=5 / 2$ ). A sensitivity test of the calculated lifetimes to the eigenvector $L S$ compositions shows that a decrease of a factor of about two of the $3 d\left({ }^{4} F\right) 4 s 4 p{ }^{4} G^{\text {o }}$ basis vector contributions (i.e., the contributions now ranging from $10 \%$ for $J=$ $11 / 2$ to $13 \%$ for $J=5 / 2$ ) to the $y^{4} G^{o}$ term increases all the calculated lifetimes by a factor of about four. Although this change is in the right direction, it is still not enough to reach a good theory-experiment agreement but itdoes show the rather high sensitivity of the computed radiative lifetimes to the change in eigenvector compositions.

In conclusion, given the good agreement between the experimental (Whaling et al. 1985) and our calculated BF values (see Figure 3 ) and the general consistencies shown in Table 1 among the available laser measurements (this work and Whaling et al. 1985; Rudolph \& Helbig 1982; Doerr et al. 1985; Xu et al. 2006), the corrected transition probabilities given in Table 2 are the ones that are recommended to the users. Moreover, this is further supported by the good agreement found between our recommended oscillator strengths and the $f$ values determined by Doerr et al. (1985; see Figure 4).

This work was supported by the National Natural Science Foundation of China (grant Nos. 11174107 and 10974066). Financial support from the Belgian FRS-FNRS is acknowledged. P.P. and P.Q. are, respectively, a Research Associate and the Research Director of this organization.

\section{REFERENCES}

Asplund, M., Grevesse, N., Sauval, J. A., \& Scott, P. 2009, ARA\&A, 47, 481 Childs, W. J., Poulsen, O., Goodman, L. S., \& Crosswhite, H. 1979, PhRvA, 19,168

Cowan, R. D. 1981, The Theory of Atomic Structure and Spectra (Berkeley, CA: Univ. California Press)

Den Hartog, E. A., Bilty, K. A., \& Lawler, J. E. 2011, JPhB, 44, 055001

Doerr, A., Kock, M., Kwiatkowski, M., Werner, K., \& Zimmermann, P. 1985, JQSRT, 33, 55

Feng, Y. Y., Jiang, L. Y., Wang, Q., et al. 2011, MNRAS, 418, 998

Feng, Y. Y., Zhang, W., Ning, L., et al. 2010, JPhB, 43, 225001

Fraga, S., Karwowski, J., \& Saxena, K. M. S. 1976, Handbook of Atomic Data (Amsterdam: Elsevier)

Larsson, J. 1994, PhyS, 49, 173

Meléndez, J., Asplund, M., Gustafsson, B., \& Yong, D. 2009, ApJL, 704, L66

Quinet, P., Palmeri, P., Biémont, E., et al. 1999, MNRAS, 307, 934

Ramírez, I., Meléndez, J., \& Asplund, M. 2014, A\&A, 561, A7

Roberts, J. R., Andersen, T., \& Sørensen, G. 1973, ApJ, 181, 587

Rudolph, J., \& Helbig, V. 1982, JPhB, 15, L599

Thorne, A. P., Pickering, J. C., \& Semeniuk, J. 2011, ApJS, 192, 11

Wang, Q., Jiang, L. Y., Jiang, H. M., et al. 2013, JPhB, 46, 045003

Whaling, W., Hannaford, P., Lowe, R. M., Biémont, E., \& Grevesse, N. 1985, A\&A, 153, 109

Xu, H. L., Jiang, Z. K., \& Lundberg, H. 2006, JOSAB, 23, 2597

Zhang, W., Feng, Y. Y., Xu, J., et al. 2010, JPhB, 43, 205005 